\title{
Spore development and nuclear inheritance in arbuscular mycorrhizal fungi
}

\author{
Julie Marleau', Yolande Dalpé1,2, Marc St-Arnaud ${ }^{1}$, Mohamed Hijri ${ }^{\text {1* }}$
}

\begin{abstract}
Background: A conventional tenet of classical genetics is that progeny inherit half their genome from each parent in sexual reproduction instead of the complete genome transferred to each daughter during asexual reproduction. The transmission of hereditary characteristics from parents to their offspring is therefore predictable, although several exceptions are known. Heredity in microorganisms, however, can be very complex, and even unknown as is the case for coenocytic organisms such as Arbuscular Mycorrhizal Fungi (AMF). This group of fungi are plant-root symbionts, ubiquitous in most ecosystems, which reproduce asexually via multinucleate spores for which sexuality has not yet been observed.

Results: We examined the number of nuclei per spore of four AMF taxa using high Z-resolution live confocal microscopy and found that the number of nuclei was correlated with spore diameter. We show that AMF have the ability, through the establishment of new symbioses, to pass hundreds of nuclei to subsequent generations of multinucleated spores. More importantly, we observed surprising heterogeneity in the number of nuclei among sister spores and show that massive nuclear migration and mitosis are the mechanisms by which AMF spores are formed. We followed spore development of Glomus irregulare from hyphal swelling to spore maturity and found that the spores reached mature size within 30 to 60 days, and that the number of nuclei per spores increased over time.

Conclusions: We conclude that the spores used for dispersal of AMF contain nuclei with two origins, those that migrate into the spore and those that arise by mitosis in the spore. Therefore, these spores do not represent a stage in the life cycle with a single nucleus, raising the possibility that AMF, unlike all other known eukaryotic organisms, lack the genetic bottleneck of a single-nucleus stage.
\end{abstract}

\section{Background}

The Arbuscular Mycorrhizal Fungi (AMF) are a group of root-inhabiting, symbiotic organisms that are widely distributed geographically and are among the most common soil fungi. AMF form symbioses with the roots of approximately $80 \%$ of all vascular plant species [1]. These fungi offer a wide variety of host benefits, the most wellknown being an increase of mineral uptake, particularly of phosphorus [2], a better drought tolerance through increased water uptake $[3,4]$ and a higher resistance to root pathogens [2]. Mycorrhizal plants also experience improved nodule function in the case of legumes [5] and

\footnotetext{
* Correspondence: Mohamed.Hijri@umontreal.ca

'Université de Montréal, Département de sciences biologiques, Institut de recherche en biologie végétale, 4101 rue Sherbrooke Est, QC, H1X 2B2, Canada

Full list of author information is available at the end of the article
}

better soil structure, due to the ability of the fungi to bind soil particles and decrease soil erosion [6].

AMF have existed unchanged morphologically for at least 460 million years, despite lacking sexual reproduction [7]. AMF are coenocytic organisms that have evolved to be multigenomic, possessing a large amount of genetic variation for ribosomal DNA [8-12], non-coding regions [13] and also for protein-coding genes $[12,14,15]$ not only between individuals, but among nuclei within an individual (one 'individual' refers here to a single spore). Reproduction occurs by asexual spores that contain hundreds or even thousands of nuclei [16,17], and these spores are the only form under which species can be identified morphologically, although AMF can be also identified using molecular markers. The ability of spores to germinate is a prerequisite for the establishment of mycorrhizal symbiosis for many AMF taxa. However, while the developmental
C Biomed Central

(c) 2011 Marleau et al; licensee BioMed Central Ltd. This is an Open Access article distributed under the terms of the Creative Commons Attribution License (http://creativecommons.org/licenses/by/2.0), which permits unrestricted use, distribution, and reproduction in any medium, provided the original work is properly cited. 
process leading to the accumulation of nuclei within spores has been recently reported to occur by the transport of numerous nuclei into the developing spore in the AMF Glomus etunicatum [18], whether or not mitosis happens within spores is not known. Fungal mitosis has been accurately described and illustrated mainly in ascomycetes and basidiomycetes and is usually intranuclear (reviewed in Aist and Morris, 1999 [19]) because the nuclear envelope remains intact, often until anaphase B. Coenocytic fungi such as zygomycetes have also an intranuclear mitosis. However, the coexistence of numerous nuclei within a common cytoplasm and mitosis phases that are not easily distinguishable causes many difficulties in studying mitosis in coenocytic fungi, although exceptions have been reported in Basidiobolus ranarum [20]. To test whether mitosis is involved during sporulation in AMF, we used mitosis inhibitors. Many mitosis inhibitors have been successfully tested on AMF. For example, Aphidicolin, which is a specific DNA polymerase $\alpha$ inhibitor that blocks the cell cycle at early $S$ phase, was used to study nuclear division in AMF during in vitro development [21]. Carbendazim, which prevents microtubule formation and inhibits mitosis in fungal cells, was also used to investigate phosphorus transport and succinate dehydrogenase activity in three AMF [22]. We chose the use of aphidicolin in our experiment because it has been shown to inhibit efficiently mitosis in AMF without any effect on spore germination [21].

To investigate the mechanisms of AMF spore formation in other taxa, and by extension, the amount of genetic material inherited by AMF offspring, we first counted the number of nuclei in live spores belonging to four different AMF taxa. All these taxa were grown in in vitro culture with Agrobacterium rhizogenes T-DNA transformed-carrot roots and were examined using confocal microscopy with a high $\mathrm{z}$-resolution for threedimensional (3D) reconstructions, which allows a direct counting of nuclei and visualization of their 3D architecture. We addressed five specific questions: 1) what is the correlation between spore diameter and the number of nuclei per spore? 2) Is there any heterogeneity in the number of nuclei among spores of similar diameter? 3) What is the mechanism of spore formation in the AMF G. irregulare? 4) What is the minimum number of nuclei required to make up a viable spore that can germinate and establish a functional symbiosis in G. irregulare? 5) Do the extraradical phases of AMF lack a single nuclear stage? These questions are of fundamental importance to our comprehension of AMF reproduction by sporulation, and their answers will bring a new level of knowledge to AMF genetics and evolution. Insight into AMF genetics is the cornerstone on which understanding the role of mycorrhizal symbiosis in nature will be based.

\section{Results}

\section{Live cell imaging of AMF spores}

We used a novel high-accuracy method for determining the number of nuclei in live spores (Figure 1, Additional Files 1 and 2) and found that in 166 live spores of $G$. diaphanum, the number of nuclei per spore ranged from 20 to 748 for diameters of 33 to $109 \mu \mathrm{m}$, respectively. Figure 2 shows the diameter of spores plotted against the number of nuclei per spore of G. diaphanum $(\mathrm{n}=166) ;$ G. irregulare $(\mathrm{n}=113) ;$ G. aggregatum $(\mathrm{n}=$ $105)$ and $G$. cerebriforme $(n=60)$. G. cerebriforme spores had the fewest nuclei per spore and were also

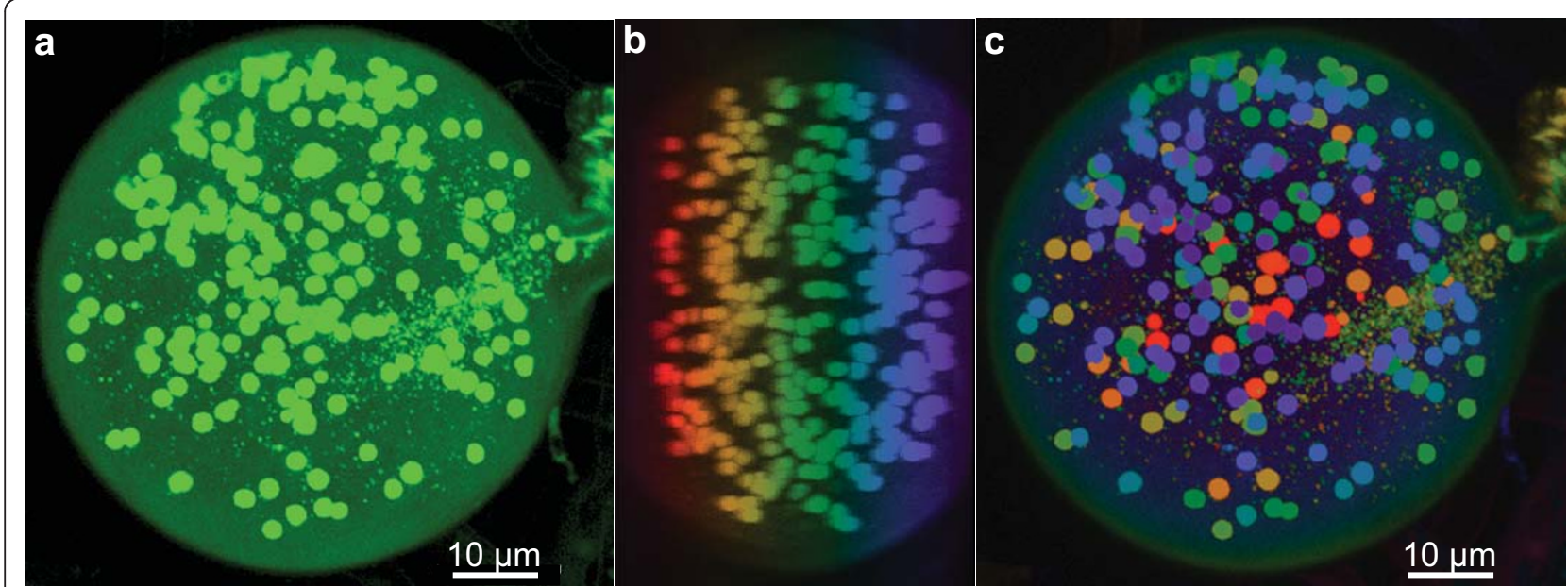

Figure 1 Live cellular imaging of AMF. Spore of G. diaphanum visualized by confocal microscope showing 208 nuclei stained with SytoGreen fluorescent dye. A, 2D ( $x, y)$ merged image with nuclei shown as green spots. B, 2D $(y, z)$ merged digital image colour-indexed on z-depth from red to violet. $\mathbf{C}, 2 \mathrm{D}(\mathrm{x}, \mathrm{y})$ merged digital image colour-indexed: red colour on the bottom and violet on the top to facilitate nuclear counting. Panels $\mathbf{A}$ and $\mathbf{C}$ are a maximum intensity projection of 260 optical sections with z-resolution of $0.15 \mu \mathrm{m}$. Scale bar represents $10 \mu \mathrm{m}$. 

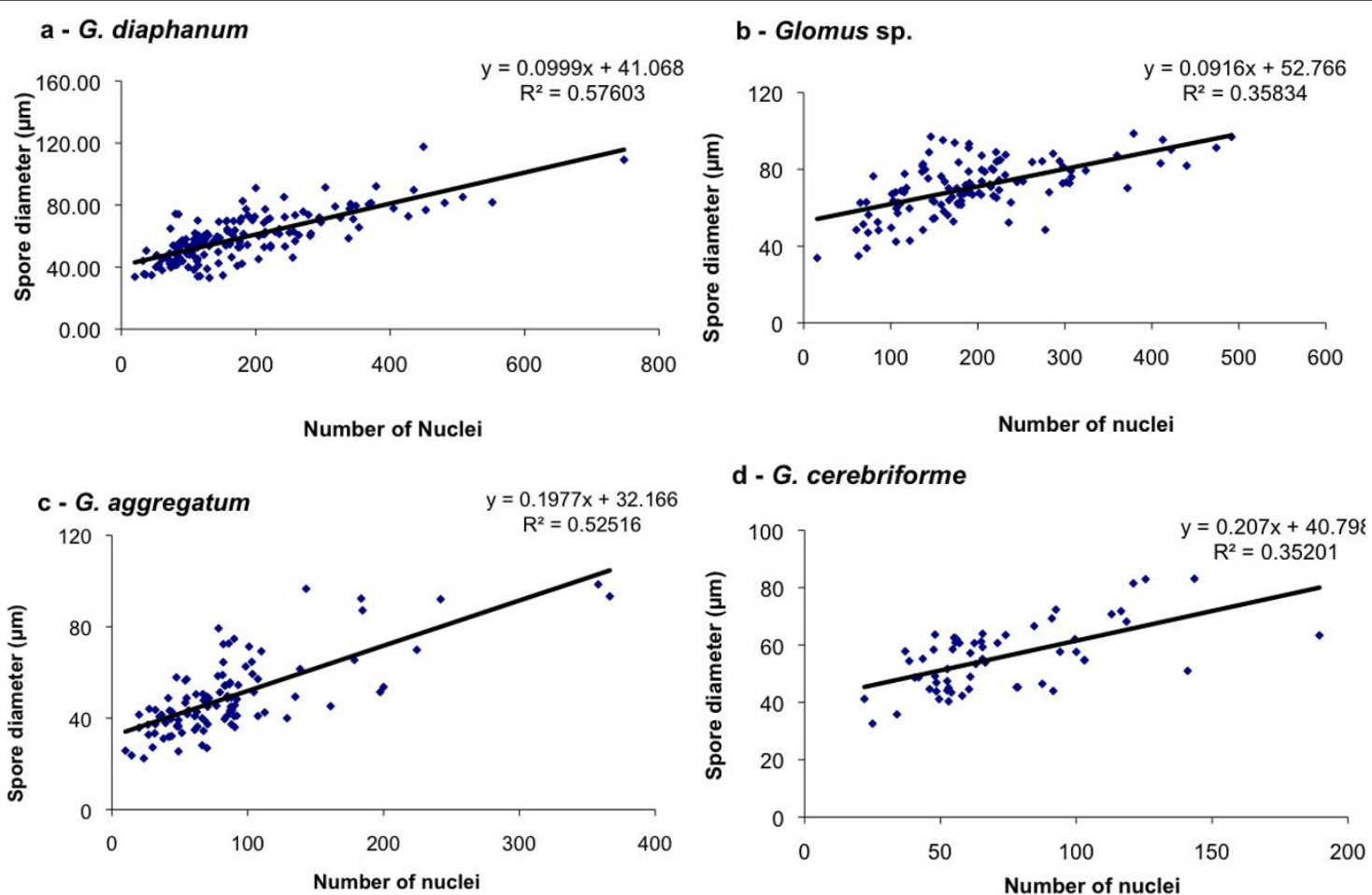

Figure 2 Number of nuclei per spore. The number of nuclei in juvenile and mature spores plotted against spore diameter of $G$. diaphanum ( $y$ $\left.=-162.53(P=0.00)+5.76 x(P=0.00), R^{2}=0.57\right)$. The diameter of spores plotted against the number of nuclei per spore. A, $G$. irregulare $(n=$ 113); B, G. aggregatum $(n=105)$ and $\mathbf{C}, G$. cerebriforme $(n=60)$. G. cerebriforme spores had the smallest number of nuclei per spore and were also the smallest in size, while $G$. diaphanum spores had the largest number of nuclei per spore. There is a positive linear relation between the number of nuclei per spore and spore diameter: $G$. irregulare $\left(y=-81.09+3.91 x, R^{2}=0.3583\right), G$. aggregatum $\left(y=-45.69+2.65 x, R^{2}=0.5252\right)$ and $G$. cerebriforme $\left(y=-21.35+1.66 x, R^{2}=0.3425\right)$. All slopes were statistically significant at $p<0.05$.

the smallest in size, while G. diaphanum spores had the greatest number of nuclei per spore. We found a positive linear relation between the number of nuclei in a given spore and its diameter for all AMF taxa: G. irregulare $\left(\mathrm{y}=-81.09+3.91 \mathrm{x}, \mathrm{R}^{2}=0.3583\right)$, G. aggregatum $(\mathrm{y}$ $\left.=-45.69+2.65 \mathrm{x}, \mathrm{R}^{2}=0.5252\right)$ and G. cerebriforme $(\mathrm{y}=$ $\left.-21.35+1.66 x, R^{2}=0.3425\right)$. All slopes were statistically significant at $p=0.05$.

\section{Heterogeneity of the number of nuclei per spores}

We also compared the number of nuclei per spore and the spore diameter of different classes for each of our four AMF taxa. We found a high heterogeneity in the number of nuclei per spore of the same diameter classes (Figure 3 and Additional File 3). For example, the number of nuclei in G. irregulare spores varied by a factor of nearly 5 in the diameter class $94-103 \mu \mathrm{m}$.

\section{Mechanism of spore formation in G. irregulare and G. diaphanum}

We used two independent, but complementary, imaging experiments to examine the nuclear inheritance process. In a two-compartmented in vitro system [23],
G. irregulare was grown on transformed carrot roots that were restricted to one compartment (proximal). Only the fungus was permitted to grow on to the second compartment (distal) containing the same media lacking the sugar and complemented with aphidicolin [17]. The formation of spores over time was monitored in the distal compartment. We found that while aphidicolin did not inhibit spore formation or hyphal growth, the number of newly produced spores was reduced by 14-fold in comparison to the control (Additional File 4). We then examined the number of nuclei per spore and the diameter of spores that were produced in the distal compartment for both aphidicolin and control treatments. The aphidicolin had no significant effect on the spore diameter $(\mathrm{W}=782, p=0.753)$, but reduced the number of nuclei per spore by $54.86 \%$ (W $=1207.5, p=$ 0.00001) (Additional File 5). On average, nuclear number per spore was $55.23 \pm 40.31 \mathrm{SE},(\mathrm{n}=22)$ and 168.30 $\pm 90.66 \mathrm{SE},(\mathrm{n}=165)$ for aphidicolin and control treatments, respectively.

To confirm this nuclear migration we next made direct observations of individual nuclei in live cells in real time. Time-lapse series taken with a confocal 


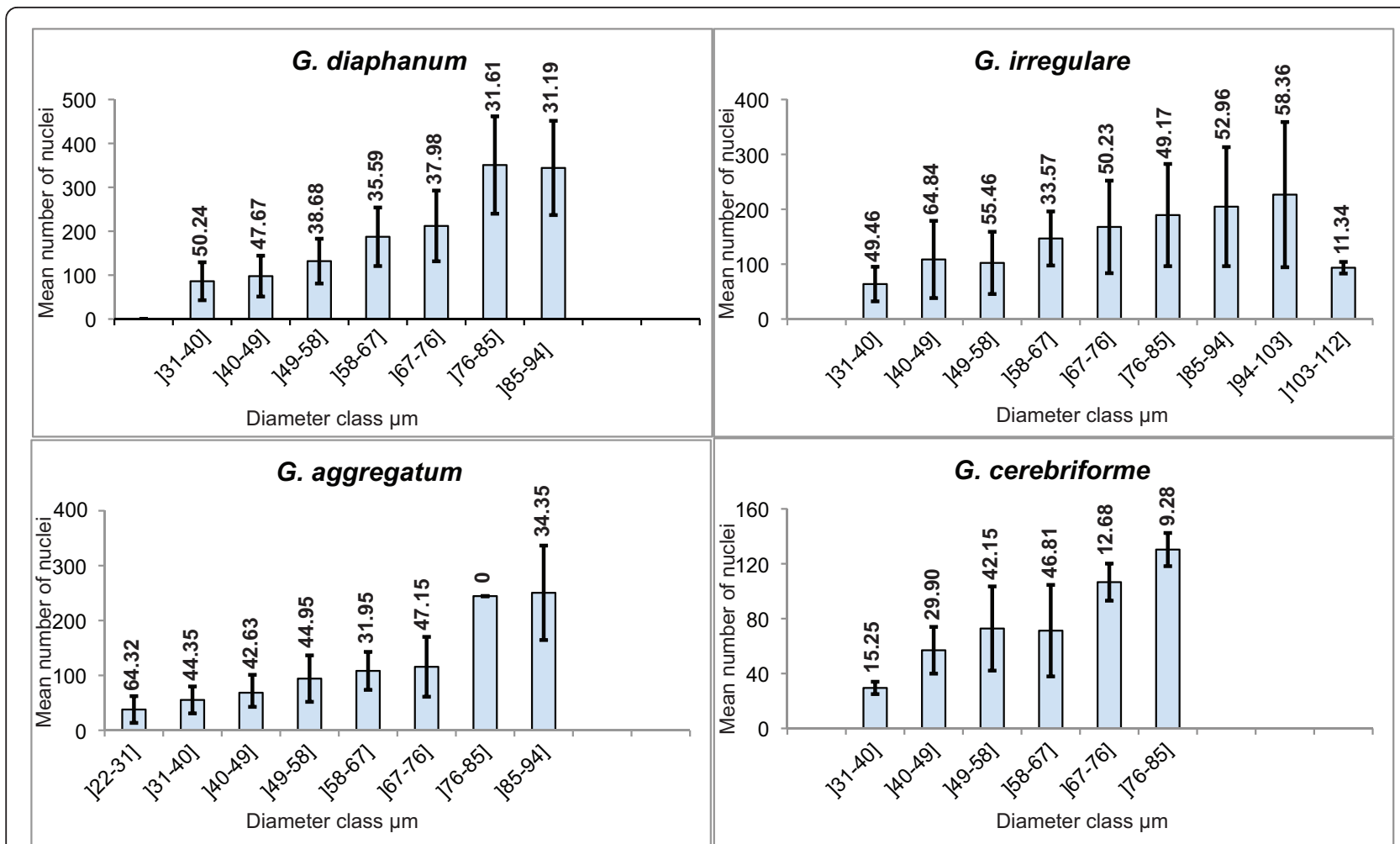

Figure 3 Heterogeneity of the number of nuclei per spore. The number of nuclei per spore compared with spore diameter grouped by classes for four AMF taxa. Coefficients of variation are shown in bold.

microscope of $G$. diaphanum spores clearly shows nuclear migration from the hypha into the developing spores (Figure 4 and Additional File 6).

\section{Spore formation over time and spore viability}

We followed spore formation of the AMF G. irregulare over time for 120 days using optical microscopy (Figure 5). We began with spore primordia (time 0) (Figure 5AB) and followed their development process at different intervals $(0,15,30,60$ and 90 days; Figure 5A-J). We found that fast spore growth occurs during the first 30 days after hyphal swelling, with a 10-fold increase in spore volume between 15 and 30 days of growth (Table 1 ) and that spore size increases slow down during the subsequent 60 days. In addition, an increase of the number of nuclei per spore over time was observed when the number of nuclei was assessed by confocal microscopy (Figure 5B, D, F, H and 5J). More importantly, while approximately $90 \%$ of the spores, from 15 days old and older, were viable as assessed by vital dye staining, only spores that were at least 30 days old were able to germinate and to regenerate an in vitro functioning colony when associated with transformed carrot roots (Table $1)$. The number of nuclei in these 30 day-old spores ranged from 65 to 222 with a mean of $153.33 \pm 46.16$ $\mathrm{SE}(\mathrm{n}=18)$. The number of nuclei in 15 day-old spores, that were viable but could not germinate, ranged from 16 to 101 with a mean of $65.09 \pm 32.1 \mathrm{SE}(\mathrm{n}=11)$. We examined the number of nuclei in mature spores that failed to germinate and observed that many of them contain no nuclei or degenerating nuclei (Additional File 7). However, some non-germinating spores had the same number of nuclei as others that were able to germinate.

\section{Nuclear stage of extraradical phase}

A careful examination of spores, runner hyphae and fine branchings from five independent extraradical symbiotic mycelia of G. diaphanum has shown that the number of nuclei per mycelium biomass changed with the morphological structure. There were more nuclei per spore than were observed in $100 \mu \mathrm{m}$ of either runner hyphae or fine branchings, a length of the same magnitude as the spore volume. However, considering their small diameter, the number of nuclei per unit volume of mycelium was significantly higher in the runner hyphae and fine branchings compared with spores (Table 2). The formation of septa was not observed in runner hyphae but was noted in $32 \%$ of the fine branchings, with numbers of nuclei varying from 0 to 18.5 per $100 \mu \mathrm{m}$ of hyphal length. Interestingly, compartments containing only a single nucleus were never observed. 

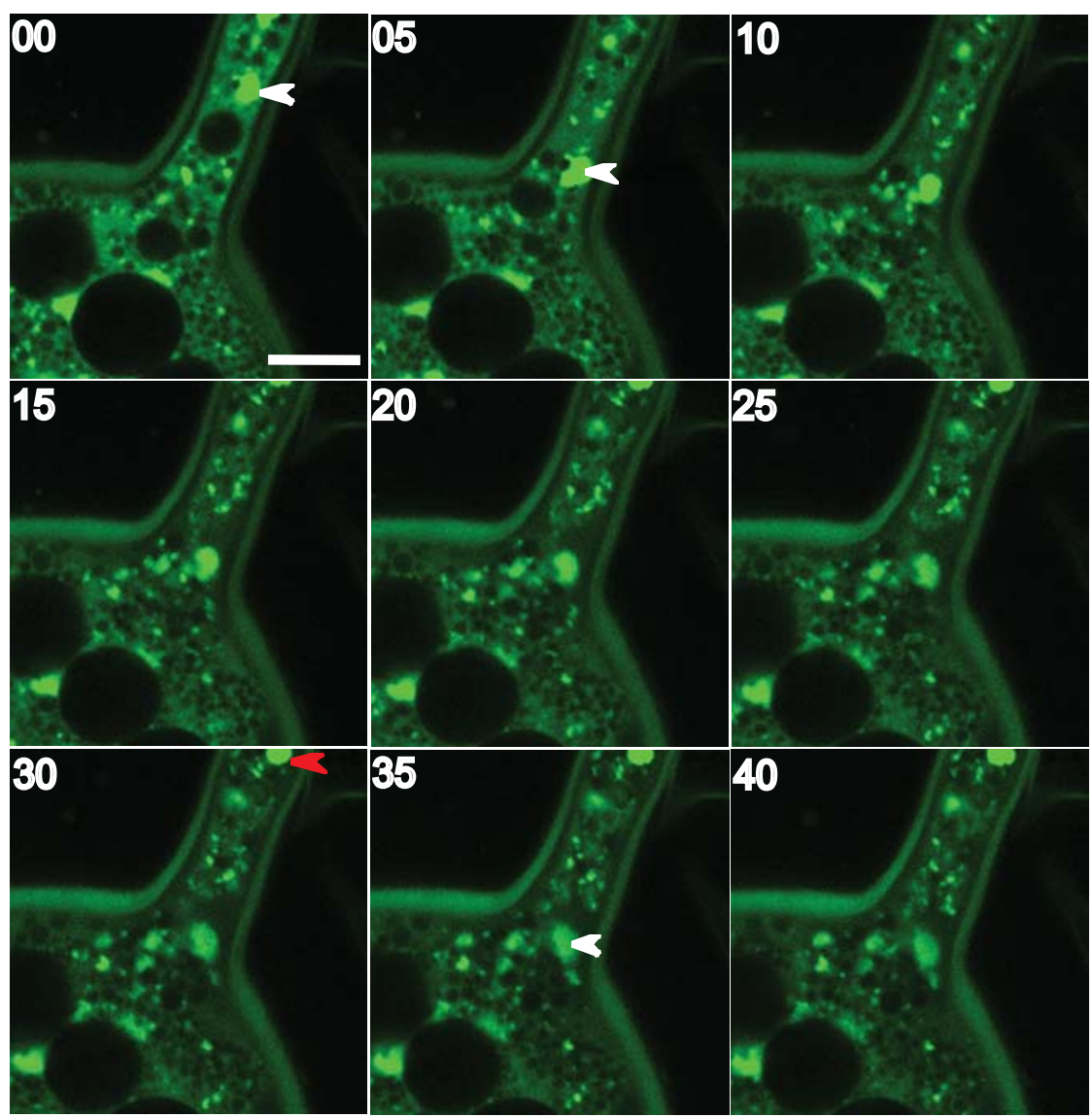

Figure 4 Time-lapse series on AMF live spores. Panels represent 5 min intervals in a time-lapse sequence monitoring nuclear migration into a developing spore of $G$. diaphanum by confocal microscopy. SytoGreen stained nuclei move unidirectionally into the spore. Images were acquired on xyt mode. Arrowheads indicate the first (white) and second (red) nucleus entering the spore. Note that some nuclei are out of focus in the xy optical section and can be difficult to visualize. Scale bar on the top-left panel represents $10 \mu \mathrm{m}$. Numbers on the top left of each panel are minutes.

\section{Discussion}

The number of nuclei in spores has been previously estimated by several methods with widely divergent results [16]. For example, using a model in which nuclei occur as a single layer around the periphery of the cytoplasm [24], the number of nuclei in Gigaspora margarita spores was estimated at about 20,000, while a different method based on digital image measurements estimated the number of nuclei per spore to be about 2,000 [17]. Here we used confocal microscopy with a high z-resolution for three-dimensional (3D) reconstructions, which allow a direct counting of nuclei and visualization of their 3D architecture in live spores. As far as we know, this is a novel rapid and high-accuracy method for determining the number of nuclei per spores in AMF. Recently, Jany and Pawlowska [18] have reconstructed 3D images of the AMF G. etunicatum using deconvolution, a computational based-method used to reduce out-of-focus fluorescence in 3D microscope images.
We found a surprisingly high heterogeneity in the number of nuclei per spore of different diameter classes. The number of nuclei in G. irregulare spores varied by about 5-fold in the diameter class 94-103 $\mu \mathrm{m}$. Clearly, the spores of the AMF used here do not have a constant number of nuclei.

We used two independent but complementary imaging experiments based on aphidicolin treatment and time-lapse live cellular imaging to examine the nuclear inheritance process in G. irregulare and G. diaphanum. We used G. irregulare in aphidicolin treatment because it is fast growing under in vitro culture. However, G. diaphanum was chosen for time-lapse experiments because its spores contained the higher number of nuclei. When tested on AMF spores, aphidicolin does not inhibit spore germination of the AMF G. margarita, but rapidly reduces the rate of hyphal growth and arrests growth after four days, owing to efficient inhibition of DNA synthesis [17]. We tested aphidicolin on 

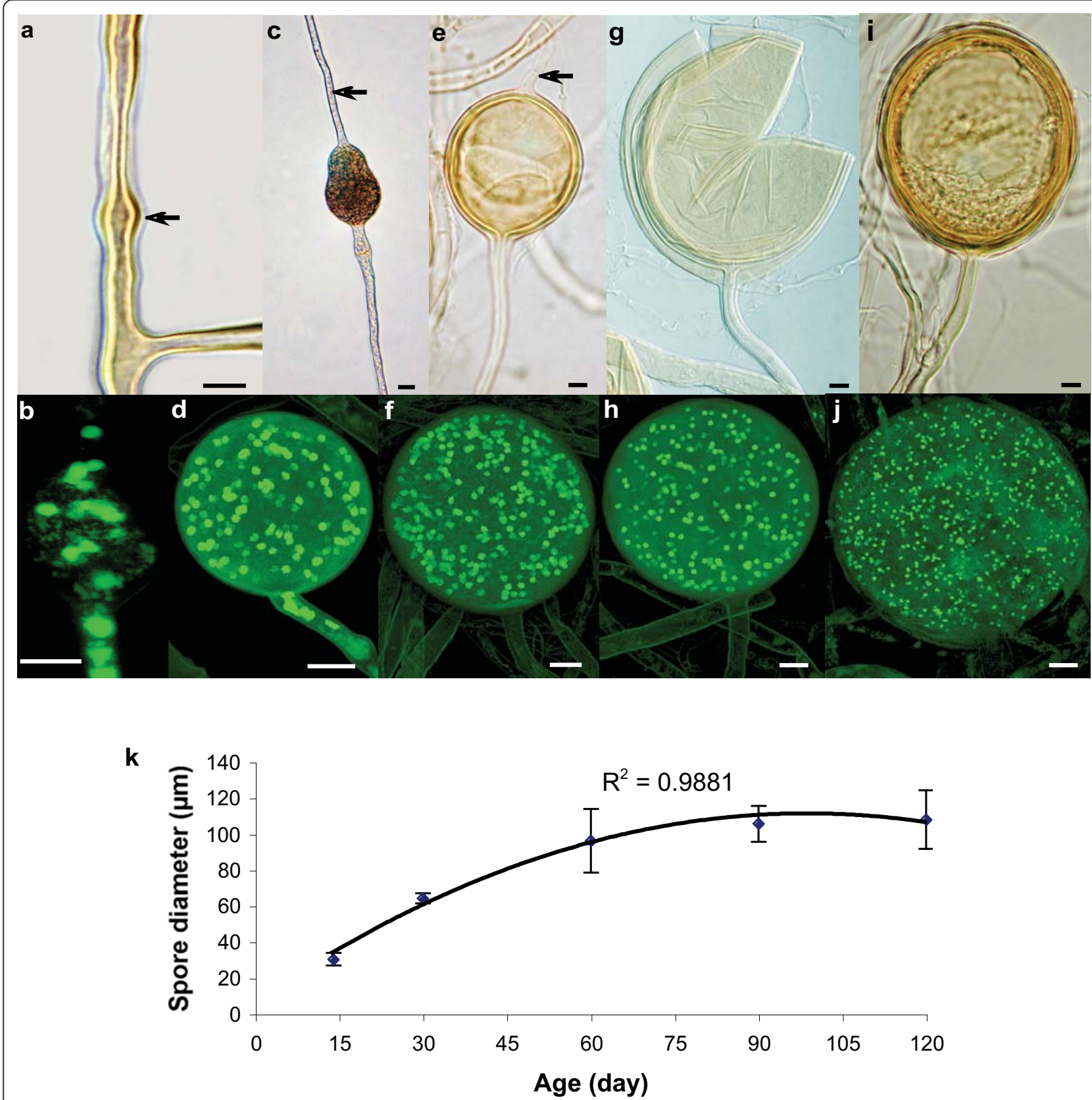

Figure $\mathbf{5}$ Spore development over time. A and $\mathbf{B}$, Intercalary swellings along the double-walled hypha give rise to a spore primordium (arrow in $\mathbf{A}$ ). $\mathbf{C}$ and $\mathbf{D}, 15$ day-old juvenile spore with subtending hypha and a septum in the mother hypha (arrow in $\mathbf{C}$ ). $\mathbf{E}$ and $\mathbf{F}$, Intercalary 30 dayold spores with subtending hypha and bi-layered spore wall. G, Crushed 60 day-old spore with subtending hypha and separating spore wall layers. The apical portion of the mother hypha has emptied, septated and detached from the spore (arrow in $\mathbf{E}$ ). $\mathbf{H}, 60$ day-old spore observed with a confocal microscope. I and $\mathbf{J}, 90$ day-old mature spore still attached to subtending hyphae. The images in panels $\mathbf{A}, \mathbf{C}, \mathbf{E}, \mathbf{G}$ and $\mathbf{I}$ were taken using a DIC optical microscope. The merged images in panels $\mathbf{B}, \mathbf{D}, \mathbf{F}, \mathbf{H}$ and $\mathbf{J}$ were taken using a confocal microscope where nuclei were stained with SytoGreen fluorescent dye. Scale bars represent $10 \mu \mathrm{m}$. K, Spore diameter plotted against spore age using a quadratic model $(\mathbf{y}=$ $\left.-0,0107 X^{2}+2,1243 X+5,9132\right)$.

yeast cells and found that no mitosis occurred in samples grown in media complemented with aphidicolin whereas optical density (DO) increased over time in samples without aphidicolin (data not shown). When spore formation was monitored over time in the distal compartment, we found that the aphidicolin did not inhibit the spore formation or the hyphal growth, but the number of newly produced spores was dramatically reduced. Aphidicolin had no significant effect on the spore diameter $\left(F_{1.88}=0.02, \mathrm{P}=0.90\right)$, but reduced the 
Table 1 A, Spore volume increase of in vitro differentiated spores of $G$. irregulare over time $(n=25)$; B, Percent spore viability and percent spore germination of in vitro differentiated spores of $G$. irregulare over time $(n=25$, for each selected age category)

\begin{tabular}{lllll}
\hline Age of spores & Mean spore diameter/radius $(\boldsymbol{\mu m})$ & Spore volume $\left(\mathbf{m m}^{\mathbf{3}}\right)$ & Viability $(\%)$ & Germination $(\%)$ \\
\hline 14 days & $30.4 / 15.2$ & 0.000015 & 90 & 0 \\
30 days & $64.2 / 32.1$ & 0.000139 & 90 & 40 \\
60 days & $96 / 48$ & 0.00046 & 90 & 38 \\
90 days & $105.7 / 52.8$ & 0.00061 & 95 & 50 \\
\hline
\end{tabular}

number of nuclei per spore by approximately three-fold $\left(F_{1.88}=15.18, \mathrm{P} \leq 0.0002\right)$. Since the nuclei observed in the spores differentiated in the distal compartment (containing aphidicolin) could not have been produced by mitosis in the newly formed spores, they must have migrated from the proximal compartment, through hyphae, into the developing spore. Considering that the average nuclear number per spore treated with aphidicolin was approximately three times less than that of the control, at least one mitotic cycle must occur that during spore development, allowing a duplication of the migrated nuclei. To confirm this nuclear migration, we made direct observations of individual nuclei in live cells in real time. Time-lapse series taken with a confocal microscope of the AMF G. diaphanum spores clearly show nuclear migration from the hypha into the developing spores. In total, we observed 534 live AMF spores of four taxa, all of which showed continuous connections between hyphal and spore cytoplasm without any apparent septa (Additional File 8). These results support the hypothesis that AMF spores are formed by a massive nuclear migration and are in agreement with results reported using G. etunicatum [18]. Interestingly, these nuclei could potentially carry different genomes, as there is considerable evidence suggesting that AMF contain populations of genetically different nucleotypes coexisting in a common cytoplasm $[12,14,15,25-28]$. In addition, genetic exchange has only recently been demonstrated to occur among AMF isolates that are genetically distinct [29]. There is as well recent evidence showing that genetic exchange and segregation in AMF significantly affects the plant response [27].

Taken together, our results clearly show that AMF spores accumulate up to thousands of nuclei by nuclear migration and mitosis. However, one potential consequence of this mechanism is the loss of the genetic diversity by drift-driven processes. During sporulation, the evolutionary process of change in the nucleotype frequencies (or genome frequencies) of a nuclear population from one generation to the next is due to the phenomenon of probability, in which pure chance events determine which nuclei within the overall nuclear population will be carried forward while others disappear. Two strategies could be envisaged to counter a potential loss of genetic diversity in AMF, either a selective sampling of certain nucleotypes from the overall population, or random selection coupled with a mechanism to ensure that spores contain adequate numbers of nuclei. Although previous models have demonstrated that hyphal fusion and nuclear exchange is an important mechanism for the maintenance of high nuclear diversity [30], this has been also reported to occur between genetically different isolate of the AMF G. intraradices [29]. Recombination has been reported to occur in AMF [31] but at low frequency and thus is likely to contribute significantly in maintaining the unusual high genetic diversity observed frequently within AMF isolates [12,28]. It has been reported that recombination repair of doublestrand DNA breaks in mitotic cells is associated with a 100-fold higher mutation rate in Saccharomyces cerevisiae [32]. In addition, S. paradoxus has a low frequency of recombination rate but maintains a completely outbreeding population structure [33].

We also wished to estimate the minimum number of nuclei to make up a viable spore (i.e., able to germinate). To address this issue, we followed spore formation of the AMF G. irregulare over time for 120 days using

Table 2 Number of nuclei in the different architectural structures forming the extraradical mycelium of G. diaphanum ( $\mathrm{n}=21$ spores, $20 \mathrm{RH}$ and $22 \mathrm{FB}$ )

\begin{tabular}{cccc}
\hline Architectural structure $^{\mathbf{1}}$ & ${\text { Diameter } \times \text { length }^{\mathbf{2}}(\boldsymbol{\mu m})}$ & Nb of nuclei & Concentration $^{\mathbf{3}}\left(\times \boldsymbol{\mu m}^{\mathbf{3}}\right)$ \\
\hline Spore & 72.6 & $282 /$ spore & $0.00147419 \mathrm{a}$ \\
RH & $6.9 \times 157.3$ & $10.3 / 100 \mu \mathrm{m}$ & $0.00306436 \mathrm{~b}$ \\
FB & $3.3 \times 131.0$ & $5.8 / 100 \mu \mathrm{m}$ & $0.00851064 \mathrm{~b}$ \\
\hline
\end{tabular}

\footnotetext{
${ }^{1}$ Structures are spores, runner hyphae (RH) and fine branching (FB).

2 Diameter (all) and length (RH, FB) or the structure portion used to count the nuclei.

${ }^{3}$ Concentrations of nuclei followed with a different letter are significantly different between structures by a Tukey-Kramer HSD test.
} 
optical microscopy. We examined the number of nuclei in mature spores that failed to germinate and observed that some had the same number of nuclei as others that were able to germinate. However, while this runs counter to the idea that spore germination requires a minimum number of nuclei, it is possible that this minimum number may vary due to other factors such as spore maturity or dormancy. Interestingly, G. etunicatum spores reached their mature size within 2-3 days [34], while G. irregulare isolate DAOM-197198 spores took 30 to 60 days to reach their mature size (Figure 5 and Additional Files 9 and 10). This is surprising because $G$. irregulare is a very fast growing AMF and therefore used as commercial inoculant sold worldwide while the G. etunicatum isolate NPI used in our laboratory has an extremely slow growth rate.

We examined various stages of the extraradical phases of G. irregulare including the symbiotic phase (running hyphae, branching hyphae and developing spores) and the resting phase (mature spores) and did not observe any structure with a single nucleus (Table 2). Although our observations were based on in vitro laboratory cultures, a single cell with a single nucleus has also never been found in nature, even though AMF are well studied in natural ecosystems. Our results provide no support for sexual reproduction or a single-nucleus stage in the life cycle of AMF, which suggests that asexual reproduction dominates in these fungi. However, demonstrations of hyphal fusion between individuals [29] and reports of recombination [13,31,35] indicate that genetic exchange and recombination also contribute to the population structure of these fungi. It has been hypothesized that auxiliary cells in Gigaspora species might represent vestiges of relict reproductive structures [36] but without evidence of karyogamy. Tommerup [37,38] has reported the formation of what was interpreted as zygospores in Gigaspora decipiens in $0.24 \%$ of hyphal contacts in a monosporal-grown mycelium, while all other contacts resulted in vegetative growth with or without anastomosis formation. Except for this case, to our knowledge gametangial formation has never been reported elsewhere despite many descriptions of the extramatrical mycelia architecture [39-42] and an extensive literature on intraradical colonization $[43,44]$.

\section{Conclusions}

We reported that AMF spores contain a high heterogeneity in the number of nuclei among sister spores and showed that massive nuclear migration and mitosis are the mechanisms contributing to the sporulation in AMF. The high levels of genetic variation within individuals combined with the large number of nuclei in AMF spores may thus be the evolutionary strategy adapted by AMF in order to reconcile their multigenomic organization with the need to remain adaptable to diverse micro-environmental changes. The finding that mitosis occurs in spore formation in AMF brings a new level of understanding of reproduction and nuclear inheritance processes in this major eukaryotic symbiosis.

\section{Methods \\ Fungal material}

The four AMF species, Glomus irregulare (isolate DAOM-197198, previously known as G. intraradices [45]), G. cerebriforme (DAOM-227022), G. aggregatum (strain 2101-sp) and G. diaphanum (DAOM-229456) used in our studies, were obtained from the Glomeromycota In vitro Collection (GINCO). These strains were co-cultured with Ri T-DNA-transformed carrot roots (Daucus carota L.). AMF strains and transformed carrot roots were cultured and maintained on a minimal (M) medium [46] solidified with $0.4 \mathrm{w} / \mathrm{v}$ Gellan gum at $25^{\circ} \mathrm{C}$.

\section{Confocal microscopy}

Arbuscular mycorrhizal fungal spores were freshly collected from plates by dissolving the Gellan gum in citrate solution [47], washed with sterile water and immediately stained with $2 \%(\mathrm{v} / \mathrm{v})$ of SytoGreen 13 live fluorescent dye (Invitrogen, Canada), for $30 \mathrm{~min}$ at room temperature and in darkness. Stained spores were mounted in an $80 \%$ glycerol solution and visualized using a Zeiss LSM 5 DUO confocal microscope equipped with Piezo (xy) stage. Specimens were imaged with an objective LCI Plan-Neofluotar $63 \times$ (NA 1.3) in water and glycerine immersion and using an argon laser at $488 \mathrm{~nm}$. For each specimen, z-stacks (approximately 150 optical sections depending on the spore size, with an interval of $0.2 \mu \mathrm{m}$ ) were recorded. Z-stacks were then merged into $2 \mathrm{D}$ images in which optical sections were colour indexed in order to facilitate manual counting of nuclei. Time-lapse experiments were performed using $(x, y, t)$ mode and 10 optical sections were collected at each $5 \mathrm{~min}$ interval for each series.

\section{Aphidicolin experiments}

Two compartment $100 \times 15 \mathrm{~mm}$ Petri dishes [23] were used in this experiment with the AMF G. irregulare. One compartment was filled with $25 \mathrm{ml}$ of $\mathrm{M}$ medium to the top level of the dividing wall, while the other compartment was filled with only $10 \mathrm{ml}$ of $\mathrm{M}$ medium lacking sugar and complemented axenically with 150 $\mu \mathrm{M}$ of aphidicolin [17] in DMSO (Sigma, Canada) after it had been autoclaved and allowed to cool to $45^{\circ} \mathrm{C}$. After solidification of the medium, an additional $1 \mathrm{ml}$ of sugarless $\mathrm{M}$ medium containing aphidicolin was deposited on the dividing wall in the sugarless compartment, placed at an angle in order to form a bridge and 
facilitate hyphal crossing between the two compartments but without continuous connection between growth media [23]. Control plates were prepared in the same conditions, except the second compartment was filled with sugarless $M$ medium complemented with an equal volume of DMSO only. G. irregulare colonized transformed carrot roots were added to the compartment containing sugar. Plates were then incubated in the dark at $25^{\circ} \mathrm{C}$ until the mycelium had crossed the dividing wall and grown on the second compartment. The cultures were examined weekly. The number of newly formed spores was counted weekly on the second compartment containing aphidicolin or DMSO as a control. After 3 months of culture, spores from this compartment were collected by dissolving the gel, rinsed with sterile and stained with SytoGreen as described above. Spores were examined as described in the confocal microscope section.

\section{Spore selection and measurement over time}

Hyphal swellings differentiated along AMF mycelium filaments on the fungal side of two-compartment plates were marked by pen on the plate bottom and their development followed under episcopic light with a dissecting microscope (Nikon SMZ 10A). Twenty-five marked 15, 30, 60 and 90 day-old spores, respectively, were recovered manually from the fungal colonies and mounted on microscopic slides in PVLG mounting medium [48]. Cross diameters of mounted spores were measured at magnification 600X (Nikon Eclipse 800), photographed (Digital camera Nikon Coolpix 950) and spore wall development sequentially described.

\section{Viability of spores}

Spore viability was estimated by the MTT 3-(4,5dimethylthiazol-yl-2,5-diphenyl-2H-tetrazolium bromide) vital stain procedure [49]. Using separate cavity slides, 25 spores were treated with MTT for 40 hours to allow maximum staining response. Treated spores were observed under dissecting microscope. Red and blue stained spores were considered viable. At 15 days old, the majority of spore primordia tested positive for viability and the level of viability remained unchanged throughout the spore maturation period (15-90 days old). The high level of viability and the uniformity of results may be attributed to the fact that spores were produced in vitro, under uniform environmental conditions and with no growth constraints. As such, the fungal material may have been less susceptible to mortality or abortion compared to spores isolated directly from soil [50].

\section{Germination potential of spores}

Twenty-five spores of each selected age category $(15,30$, 60, 90 days) were extracted from two-compartment in vitro cultures, deposited on $\mathrm{H}_{2} \mathrm{O}$ agar medium ( $\mathrm{pH}$ 6.0) and incubated for a maximum of 30 days at $27^{\circ} \mathrm{C}$ in darkness. Spores bearing germinating hyphae longer than $150 \mu \mathrm{m}$ were considered germinated. Thirty dayold spores already showed a $40 \%$ germination potential and the capacity to regenerate an in vitro functioning colony when associated with transformed carrot roots. This germination potential, whatever the level of spore maturity, never reached more than $50 \%$ of the spores, even in those considered viable according to the MTT staining test. The 30 days incubation period chosen may not have been sufficient to demonstrate the full germination capability. However, most of the spore germination observed occurred in the first 3-6 days after incubation, indicating a sufficient maturity to undergo the germination process.

\section{AMF extraradical mycelium}

The extraradical symbiotic mycelium of AMF exhibits three distinguishable morphological structures: largediameter, relatively unbranched, thick-walled hyphae called runner hyphae (RH) [40], small-diameter thinwalled branched hyphae called arbuscule-like structures [51] or fine branching (FB) [52] and spores. To assess for the presence of uninucleate structures, the mycelium of G. diaphanum was carefully examined. Mycelia samples were harvested from five different plate cultures by dissolving the gellan gum in citrate buffer [47], washed with sterile water and immediately stained with $10 \mu \mathrm{M}$ SytoGreen 13 for $60 \mathrm{~min}$ at $35^{\circ} \mathrm{C}$ in the dark. Stained mycelia were mounted in $50 \%$ glycerol solution on a glass plate and 20-22 RH, FB and spores were randomly chosen and visualized using a Zeiss confocal microscope as described above. The nuclei were counted and the volume was calculated from the diameter (for all structures) and length (for RH and FB).

\section{Statistical analyses}

Statistical analyses were conducted using the using JMP 7.0 and Statgraphics Plus v3 statistical software. Since no transformation produced a normal distribution of the residuals, Kruskal-Wallis non-parametric one-way analysis of variance by ranks were conducted to determine the effect of aphidicolin on spore diameter and number of nuclei per spore, and to compare the number of nuclei between morphological structures of G. diaphanum symbiotic extraradical mycelia, while a posteriori comparisons between means were done using Tukey-Kramer honestly significant difference tests. In order to determine the relationship between the number of nuclei per spore and the diameter of spores, a linear regression model $(y=a x+b)$ was fitted to the number of nuclei (y) with respect to spore diameter $(x)$ for each AMF species. 


\section{Additional material}

Additional file 1: Example of a z-stack series of Glomus diaphanum live spore observed with a confocal microscope. Nuclei were visualized as large green spots with SytoGreen fluorescent dye, while mitochondria are shown as small green spots. The movie was acquired at 1 frame every $0.15 \mu \mathrm{m}$ for a total of 260 frames and displayed at a rate of 5 frames/sec.

Additional file 2: 3D reconstruction of 260 optical sections with zresolution of $0.15 \mu \mathrm{m}$, showing a full turn of $\mathrm{G}$. diaphanum live spore with nuclei color indexed. The movie is displayed at a rate of 5 frames/sec.

Additional file 3: Merged image of 100 optical sections of sister spores of $G$. diaphanum showing heterogeneity of nuclear content. Nuclei were stained with SytoGreen fluorescent dye. Scale bar represents $47.62 \mu \mathrm{m}$.

Additional file 4: The mean number of newly produced spores plotted against time (days) of G. irregulare treated with aphidicolin diluted in DMSO or DMSO alone as a control on six replicates each Red and blue curves represent aphidicolin and control treatments, respectively.

Additional file 5: The number of nuclei per spore plotted against the diameter of spores of $G$. irregulare treated with aphidicolin (red squares, $n=22$ ) and the control (blue triangles, $n=68$ ).

Additional file 6: Time-lapse series on $G$. diaphanum live spore Nuclei were visualized as large green spots with SytoGreen fluorescent dye, while mitochondria were stained with MitoTracker and are shown as small red spots. The movie was acquired at 1 frame every 5 min for a total of $90 \mathrm{~min}$ and displayed at a rate of 5 frames $/ \mathrm{sec}$.

Additional file 7: Merged image of 150 optical sections of nongerminating spore of $G$. diaphanum showing nuclei in a degenerating phase. Nuclei were stained with SytoGreen fluorescent dye. Scale bar represents $19.71 \mu \mathrm{m}$.

Additional file 8: G. diaphanum spore observed with confocal microscope where nuclei were visualized by SytoGreen fluorescent dye (green spots) showing direct connection of spore and the subtending hyphal cytoplasm (arrows). A, xy focal plane; $B, y z$ projection; and C, xz projection. Scale bar in panel A represents 17.93 $\mu \mathrm{m}$.

Additional file 9: AMF spore development of G. irregulare over time, viability and germination rates.

Additional file 10: Cell wall structure of $G$. intraradices spores of different ages. A, Open 30 day-old spore with subtending hypha, outside hyaline spore wall and inside pale yellow pigmented spore wall. $\mathrm{B}$, Closer view of 60 day-old spore wall with bi-layered outer walls and laminated inner wall. C, Walls of 90 day-old mature spore with bi-layered outer wall and multi-laminated inner wall. Images were taken with DIC optical microscope. Scale bars represent $20 \mu \mathrm{m}$.

\section{List of abbreviations}

AMF: Arbuscular Mycorrhizal Fungi; 3D: three-dimensional; DAPI: 4',6diamidino-2-phenylindole; MTT: 3-(4,5-dimethylthiazol-yl-2,5-diphenyl-2Htetrazolium bromide); $\mathrm{RH}$ : runner hyphae; FB: fine branching; DMSO: Dimethyl sulfoxide; DAOM: National Mycological Herbarium.

\section{Acknowledgements}

This work was supported by NSERC discovery grant to MH. We thank the Canada Foundation for Innovation (CFI) grant to $\mathrm{MH}$ for the confocal microscopy facility and FQRNT for award of a scholarship to JM. We thank Dr I.R. Sanders for allowing part of this work to be conducted in his lab at the University of Lausanne. We also thank K. Coquart for technical assistance, Dr R. Lahlali for assistance on statistics, Dr D. Morse, Dr. C. Cameron and Dr. R. Maranger for comments and discussions.

\section{Author details}

${ }^{1}$ Université de Montréal, Département de sciences biologiques, Institut de recherche en biologie végétale, 4101 rue Sherbrooke Est, QC, H1X 2B2, Canada. ${ }^{2}$ Agriculture and Agri-Food Canada, 960 Carling Ave. Ottawa, On, K1A 0C6, Canada.

\section{Authors' contributions}

JM performed confocal imaging and aphidicolin experiments, analyzed data and contributed in writing the manuscript. YD performed spore

development experiments and contributed in writing the manuscript. MSA designed experiments on AMF extraradical phases and analyzed the data. $\mathrm{MH}$ (corresponding author) designed experiments, evaluated the data, coordinated the research project, constructed the figures and wrote the manuscript. All of the authors read and approved the final manuscript.

Received: 20 August 2010 Accepted: 24 February 2011

Published: 24 February 2011

\section{References}

1. Smith SE, Read DJ: Mycorrhizal symbiosis. Amsterdam: Academic press, 3 2008.

2. Helgason T, Fitter A: The ecology and evolution of the arbuscular mycorrhizal fungi. Mycologist 2005, 19(3):96-101.

3. Augé RM: Water relations, drought and vesicular-arbuscular mycorrhizal symbiosis. Mycorrhiza 2001, 11(1):3-42.

4. Augé RM: Arbuscular mycorrhizae and soil/plant water relations. Can J Soil Sci 2004, 84(4):373-381

5. Barea JM, Azcon R, Azcon-Aguilar C: Mycorrhizosphere interactions to improve plant fitness and soil quality. Antonie Van Leeuwenhoek 2002, 81(1-4):343-351.

6. Rillig MC, Mummey DL: Mycorrhizas and soil structure. New phytol 2006, 171(1):41-53.

7. Redecker D, Kodner R, Graham LE: Glomalean fungi from the Ordovician Science 2000, 289(5486):1920-1921.

8. Sanders IR, Alt M, Groppe K, Boller T, Wiemken A: Identification of ribosomal DNA polymorphisms among and within spores of the Glomales: application to studies on the genetic diversity of arbuscular mycorrhizal fungal communities. New Phytol 1995, 130(3):419-427.

9. Lloyd-Macgilp SA, Chambers SM, Dodd JC, Fitter AH, Walker C, Young JPW: Diversity of the ribosomal internal transcribed spacers within and among isolates of Glomus mosseae and related mycorrhizal fungi. New Phytol 1996, 133(1):103-111.

10. Hijri M, Hosny $M$, van Tuinen D, Dulieu H: Intraspecific ITS polymorphism in Scutellospora castanea (Glomales, Zygomycota) is structured within multinucleate spores. Fungal Genet Biol 1999, 26(2):141-151.

11. Lanfranco $L$, Delpero $M$, Bonfante P: Intrasporal variability of ribosomal sequences in the endomycorrhizal fungus Gigaspora margarita. Mol Ecol 1999, 8(1):37-45.

12. Kuhn G, Hijri M, Sanders IR: Evidence for the evolution of multiple genomes in arbuscular mycorrhizal fungi. Nature 2001, 414(6865):745-748

13. Pawlowska TE, Taylor JW: Organization of genetic variation in individuals of arbuscular mycorrhizal fungi. Nature 2004, 427(6976):733-737.

14. Hijri M, Sanders IR: Low gene copy number shows that arbuscular mycorrhizal fungi inherit genetically different nuclei. Nature 2005, 433(7022):160-163.

15. Hijri M, Niculita $H$, Sanders IR: Molecular characterization of chromosome termini of the arbuscular mycorrhizal fungus Glomus intraradices (Glomeromycota). Fungal Genet Biol 2007, 44(12):1380-1386.

16. Viera A, Glenn MG: DNA content of vesicular-arbuscular mycorrhizal fungal spores. Mycologia 1990, 82(2):263-267.

17. Bécard G, Pfeffer PE: Status of nuclear division in arbuscular mycorrhizal fungi during in vitro development. Protoplasma 1993, 174(1-2):62-68.

18. Jany $J$, Pawlowska TE: Multinucleate spores contribute to evolutionary longevity of asexual glomeromycota. Am Nat 175(4):424-435.

19. Aist JR, Morris R: Mitosis in Filamentous Fungi: HowWe Got WhereWe Are. Fungal Genetics and Biology 1999, 27:1-25

20. Tanaka K: Mitosis in the Fungus Basidiobolus ranarum as Revealed by Electron Microscopy. Protoplasma 1970, 70:423-440.

21. Bécard G, Pfeffer PE: Status of nuclear division in arbuscular mycorrhizal fungi during in vitro development. Protoplasma 1993, 174:62-68. 
22. Kling $M$, Jakobsen I: Direct application of carbendazim and propiconazole at field rates to the external mycelium of three arbuscular mycorrhizal fungi species: effect on 32P transport and succinate dehydrogenase activity. Mycorrhiza 1997, 7:33-37.

23. St-Arnaud M, Hamel C, Vimard B, Caron M, Fortin JA: Enhanced hyphal growth and spore production of the arbuscular mycorrhizal fungus Glomus intraradices in an in vitro system in the absence of host roots. Mycol Res 1996, 100:328-332.

24. Burggraaf AJP, Beringer JE: Nuclear division and VA-mycorrhizal in-vitro culture. In Mycorrhizae in the next decade: Practical applications and research priorities; Proceedings of the 7th Norh American conference on mycorrhizae. Edited by: Sylvia DM, Hung LL, Graham JH. Gainesville: University of Florida; 1987:190.

25. Cardenas-Flores A, Draye X, Bivort C, Cranenbrouck S, Declerck S: Impact of multispores in vitro subcultivation of Glomus sp. MUCL 43194 (DAOM 197198) on vegetative compatibility and genetic diversity detected by AFLP. Mycorrhiza 20(6):415-425.

26. Croll D, Giovannetti M, Koch AM, Sbrana C, Ehinger M, Lammers PJ, Sanders IR: Nonself vegetative fusion and genetic exchange in the arbuscular mycorrhizal fungus Glomus intraradices. New Phytol 2008.

27. Angelard C, Colard A, Niculita-Hirzel H, Croll D, Sanders IR: Segregation in a mycorrhizal fungus alters rice growth and symbiosis-specific gene transcription. Curr Biol 2010, 20(13):1216-1221.

28. Boon E, Zimmerman E, Lang BF, Hijri M: Intra-isolate genome variation in arbuscular mycorrhizal fungi persists in the transcriptome. J Evol Biol 2010, 23(7):1519-1527.

29. Croll D, Giovannetti M, Koch AM, Sbrana C, Ehinger M, Lammers PJ, Sanders IR: Nonself vegetative fusion and genetic exchange in the arbuscular mycorrhizal fungus Glomus intraradices. New Phytol 2009, 181:924-937.

30. Bever JD, Wang M: Arbuscular mycorrhizal fungi: hyphal fusion and multigenomic structure. Nature 2005, 433:E3-4.

31. Croll D, Sanders IR: Recombination in Glomus intraradices, a supposed ancient asexual arbuscular mycorrhizal fungus. BMC Evol Biol 2009, 9:13.

32. Strathern JN, Shafer BK, McGill CB: DNA synthesis errors associated with double-strand-break repair. Genetics 1995, 140:965-972.

33. Tsai IJ, Burt A, Koufopanou V: Conservation of recombination hotspots in yeast. Proc Natl Acad Sci USA 107(17):7847-7852.

34. Jany $\mathrm{J}$, Pawlowska TE: Multinucleate spores contribute to evolutionary longevity of asexual glomeromycota. Am Nat 2010, 175(4):424-435.

35. den Bakker HC, Vankuren NW, Morton JB, Pawlowska TE: Clonality and recombination in the life history of an asexual arbuscular mycorrhizal fungus. Mol Biol Evol 2010, 27:2474-2486.

36. Morton JB, Benny GL: Revised classification of arbuscular mycorrhizal fungi (zygomycetes): a new order, glomales, 2 new suborders, Glomineae and Gigasporineae, and 2 new families, Acaulosporaceae and Gigasporaceae, with an emendation of Glomaceae. Mycotaxon 1990, 37:471-491.

37. Tommerup IC: The vesicular-arbuscular mycorrhizas. In Advance in plant pathology. Volume 6. Edited by: Ingram DS, William PH. San Diego: Academic Press; 1988.

38. Tommerup IC, Sivasithamparam K: Zygospores and asexual spores of Gigaspora decipiens, an arbuscular mycorrhizal fungus. Mycol Res 1990, 94:897-900.

39. Giovannetti M, Fortuna P, Citernesi AS, Morini S, Nuti MP: The occurrence of anastomosis formation and nuclear exchange in intact arbuscular mycorrhizal networks. New Phytol 2001, 151(3):717-724.

40. Friese CF, Allen MF: The spread of VA mycorrhizal fungal hyphae in the soil: inoculum types and external hyphal architecture. Mycologia 1991, 83(4):409-418.

41. Giovannetti M, Azzolini D, Citernesi AS: Anastomosis formation and nuclear and protoplasmic exchange in arbuscular mycorrhizal fungi. Appl Environ Microbiol 1999, 65(12):5571-5575.

42. Bago B, Azcón-Aguilar C, Piché Y: Architecture and developmental dynamics of the external mycelium of the arbuscular mycorrhizal fungus Glomus intraradices grown under monoxenic conditions. Mycologia 1998, 90(1):52-62.

43. Dodd JC, Boddington CL, Rodriguez A, Gonzalez-Chavez C, Mansur I: Mycelium of Arbuscular Mycorrhizal fungi (AMF) from different genera: form, function and detection. Plant Soil 2000, 226:131-151.
44. Dickson S, Smith FA, Smith SE: Structural differences in arbuscular mycorrhizal symbioses: more than 100 years after Gallaud, where next? Mycorrhiza 2007, 17(5):375-393

45. Sokolski S, Dalpé Y, Séguin S, Khasa D, Lévesque CA, Piché Y: Conspecificity of DAOM 197198, the model arbuscular mycorrhizal fungus, with Glomus irregulare: molecular evidence with three proteinencoding genes. Botany 2010, 88:829-838.

46. Bécard G, Fortin JA: Early events of vesicular-arbuscular mycorrhiza formation on Ri T-DNA transformed roots. New Phytol 1988, 108(2):211-218.

47. Doner LW, Bécard G: Solubilization of gellan gels by chelation of cations. Biotechnol Tech 1991, 5(1):25-28.

48. Omar MB, Bolland L, Heather WA: Permanent mounting medium for fungi. Stain Technol 1978, 53(5):293-294.

49. An ZQ, Hendrix JW: Determining viability of Endogonaceous spores with a vital stain. Mycologia 1988, 80(2):259-261.

50. Meier R, Charvat I: Reassessment of tetrazolium bromide as a viability stain for spores of vesicular-arbuscular mycorrhizal fungi. Am J Bot 1993, 80(9):1007-1015

51. Mosse B: Observations on the extra-matrical mycelium of a vesiculararbuscular endophyte. Trans Br Mycol Soc 1959, 42:439-448.

52. Juge $C$, Coughlan AP, Fortin JA, Piché Y: Growth and branching of asymbiotic, pre-symbiotic and extraradical AM fungal hyphae: clarification of concepts and terms. In Advances in mycorrhizal science and tehnology. Edited by: Khasa DP, Piché Y, Coughlan AP. Ottawa: NRC research press; 2009:39-50.

doi:10.1186/1471-2148-11-51

Cite this article as: Marleau et al:: Spore development and nuclear inheritance in arbuscular mycorrhizal fungi. BMC Evolutionary Biology 2011 11:51.

\section{Submit your next manuscript to BioMed Central and take full advantage of:}

- Convenient online submission

- Thorough peer review

- No space constraints or color figure charges

- Immediate publication on acceptance

- Inclusion in PubMed, CAS, Scopus and Google Scholar

- Research which is freely available for redistribution

Submit your manuscript at www.biomedcentral.com/submit
C Biomed Central 